\title{
Special features of transparent, porous alumina xerogels
}

\author{
V SARASWATI* \\ Materials Development Laboratory, Indira Gandhi Centre for Atomic Research, \\ Kalpakkam 603 102, India \\ *Present address: Defence Metallurgical Research Laboratory, Kanchanbagh, Hyderabad \\ 500258 , India \\ MS received 11 January 1988; revised 19 March 1988
}

\begin{abstract}
The process of gelation through chemical polymerization is described for the preparation of transparent alumina. The nature of disorder in the transition phases of alumina is discussed using the results of XRD, IR, optical absorption and NMR. The microstructures in the monolithic porous xerogels are observed to be fractals arising due to instabilities while drying. Phase separation noticed in copper and chromium-doped xerogels is attributed to fluctuations in polymer density.
\end{abstract}

Keywords. Transparent alumina; porous alumina; xerogel; fractals.

\section{Introduction}

Solgel process is commonly used for producing fine size powders and homogeneous multicomponent materials (Roy 1956). In recent years it is in vogue again because molecules can be chemically polymerized favouring the production of bulk glass, films and fibres at lower temperatures. Apart from saving energy, it also opens up novel scientific and technological possibilities. The hydrolysis, condensation and polymerization of metal alkoxides yield inorganic polymers with oxide skeleton framed by hydroxyl and organic groups. On heating, an oxide network is left behind. Silica thus prepared was almost similar to the glass prepared by melt quench technique (Mackenzie 1982) although some differences were reported (Yoldas 1982).

Preparation of transparent alumina monoliths and their properties are presented here. The influence of the microstructure, viz the ultrafine particle size, porosity and unstable aggregation patterns have been studied. The coloured alumina monoliths were obtained by the interaction of transition metal ion with the gel. As the coloured monoliths were transparent it was possible to carry out optical measurements to ascertain the valence state and coordination number of the transition metal ion (Saraswati and Ramarao 1987), which were earlier determined from ESR and susceptibility data (Pott and McNicol 1970). These are of value in the usage of gamma alumina as catalyst supports.

\section{Preparation of the monoliths}

The gelation process can be carried out using different precursor solutions. Schematically a solgel process can be written as follows:

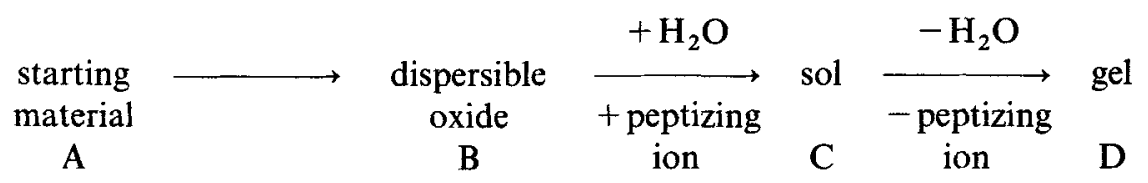


A could be a solution of metallic salt or colloidal sol. Step A to B may or may not involve extensive chemical processing depending on the material. Sometimes a dilute acid is used apart from water to peptize and get a clear stable sol C. Mixture of sols may be used at $\mathrm{C}$ to get multicomponent systems. The processing between $\mathrm{C}$ and $\mathrm{D}$ can be modified to obtain particles, microspheres etc. Sol to gel transition $\mathrm{C} \rightarrow \mathrm{D}$ is effected by dehydration, either by heating or by adding an anhydrous immiscible organic solvent like 2-ethyl hexanol. On concentration, with reduction in the interparticle distance, gelation through electrolytic effects starts. The nature of the electrolyte and its concentration influence the gelling time and volume. The dehydrated gel is a physical aggregate. It is crystalline and usually consists of particles of size about a few hundred angstroms.

Another route to gelation is through the sol of polymerizable alkoxides. There are fundamental differences in the gelation when $\mathrm{A}$ is a solution of polymerizing species like metal organic compounds. The gelation occurs as a result of polymerization, branching and crosslinking of molecular chains through chemical reaction. The nature and kinetics of these reactions determine the properties of the gel and the resultant inorganic polymer. Controlling of hydrolysis is imperative, otherwise one will end up with fine oxide powder as from colloidal sols. Polymerization takes place only when soluble polymerizable species are present in the liquid for sufficient time. This is achieved by making use of mutual solvents like alcohols and controlling the molar ratio of alkoxide to water to catalyst, which can be an acid or base. This route has opened up the possibility of obtaining transparent bulk gel, film or fibre just by controlling the alkoxide to water and acid ratio (Sakka 1985). Composites at the molecular level have been prepared (Hoffman et al 1984). New abrasives and cermets have also been produced (Roy and Roy 1984). It is significant that the control and manipulation of material character are at the molecular level.

Pseudoboehmite gels were prepared by hot hydrolysis of aluminium isopropoxide (Yoldas 1975). Hydrolysis was accomplished by mixing isopropoxide in isopropanol, water and hydrochloric acid and refluxing the same at $80 \mathrm{C}$ in water bath for 2 to $6 \mathrm{~h}$. A translucent or clear sol was obtained depending on the water and acid content. The clear sol kept in an oven at $80 \mathrm{C}$ gelled within 20 to $40 \mathrm{~h}$. Transparent gels were obtained when the alkoxide-water-acid ratio was as $1: 100: 03$. Pieces of gels were taken out dried and tested.

The hydrolysis and condensation reactions can be written as

$$
\mathrm{Al}(\mathrm{OR})_{3}+2 \mathrm{H}_{2} \mathrm{O} \longrightarrow \mathrm{AlOOH}+3 \mathrm{ROH} .
$$

The monohydroxide, boehmite, can react with water to produce the trihydroxide $\mathrm{AlOOH}+\mathrm{H}_{2} \mathrm{O} \longrightarrow \mathrm{Al}(\mathrm{OH})_{3}$.

The formation of trihydroxide (bayerite) was prevented by increasing the temperature of hydrolysis to $80 \mathrm{C}$. Polymerization takes place as

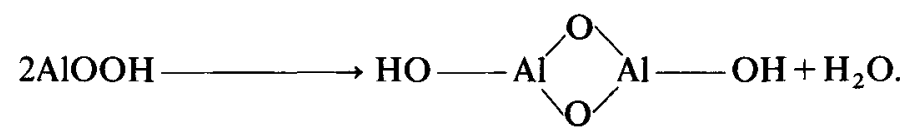

Polymerization is a step growth process with a slow build-up of the molecular weight (Uhlmann et al 1984). The dimers interact with monomers to produce trimers and so on. Linear polymers form with less water and more acid whereas 
more water facilitates crosslinking and yields a bulk gel. The gel was dried in a flat dish to obtain monoliths. The drying rate was controlled to obtain crackfree gels. Some of the monoliths obtained are shown in figure 1 .

Transition metals were introduced as pure powder, wire or foil in the wet gel which interacts with it probably due to its high reactivity and gels get coloured owing to the formation of complex hydroxides at the surface. The colour depends on the metal ion, its concentration and its valence state. Doping with iron gave a yellow colour, copper green or blue and chromium green. When the dopant concentration was $<1000 \mathrm{ppm}$ the monoliths were colourless. The concentration was estimated spectrochemically.

\section{Polymorphic transformations}

DTA. TG loss and IR measurements in the powder confirmed the formation of the monohydroxide, boehmite. The polymorphic transformations to alumina phases occur as

$$
\text { boehmite } \stackrel{350 \mathrm{C}}{\longrightarrow} \gamma \stackrel{900 \mathrm{C}}{\longrightarrow} \mathrm{\delta} \stackrel{1150 \mathrm{C}}{\longrightarrow} \alpha \mathrm{Al}_{2} \mathrm{O}_{3} .
$$

The transition takes place at the temperatures shown when it is held constant for $10 \mathrm{~h}$ or more. For fast heating as in DTA or when the hold is short. transitions are at higher temperatures. At the transition, structural changes occur due to rearrangement of oxygen atoms.

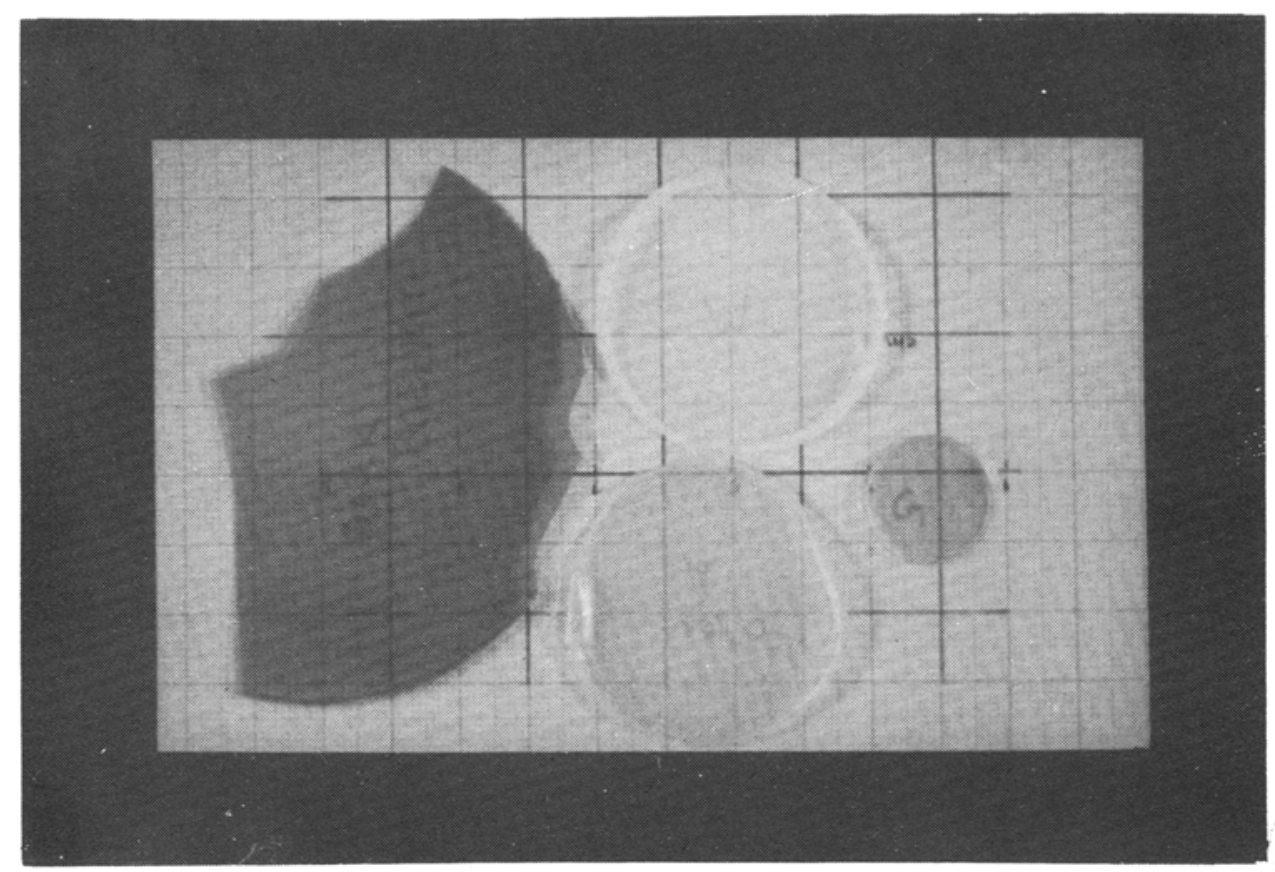

Figure 1. Alumina monolithic xerogels. a. $\mathrm{Cr} \quad(3 \mathrm{wt} \% \mathrm{l}$ deped boehmite (dark green), b. undoped alumina, c. Fe $(0.98 \mathrm{wt} \%)$ doped $;$-alumina (yellow) and d. $\mathrm{Cu}$ (1). $6 \mathrm{wt} \%$ ) doped $;$-alumina (green). 


\section{Structure and disorder}

The structural evolution of boehmite on heating was discussed in an earlier paper (Saraswati et al 1987). On dehydration. the oxygen atoms in boehmite rearrange to cubic close-packed in $;$, tetragonal in $\delta$ and hexagonal close-packed in $\alpha$ phase. Compared to the sharp and intense X-ray diffraction peaks to the $\alpha$ phase powder. broad diffuse and weak peaks were observed for boehmite, $;$ and $\dot{\delta}$ phases of alumina. The line broadening could arise due to the ultrafine size of particles, which was $8 \mathrm{~nm}$ when measured from TEM and surface area analysis. Further, when smaller wavelength $\mathrm{MoK}_{x}$ radiation $(i=0.07 \mathrm{~nm})$ was used instead of $\mathrm{CuK}_{\alpha}$ radiation $(\lambda=0 \cdot 154 \mathrm{~nm})$, an increased number of reflections were observed and the calculated lattice parameters agreed with the earlier work of Lippens and deBoer (1964). Boehmite structure consists of layers of $\mathrm{AlO}_{6}$ octahedra separated by hydrogen-bonded interlayer water molecules (Wilson 1979). The $d$ spacing of X-ray reflection at 002 depends on the amount of water. On heating at $350 \mathrm{C}$ the $;$ alumina is formed which bears a topotactic relation to boehmite. The oxygen sublattice is considered to be fairly ordered (cubic close packed) whereas tetrahedral Al lattice is strongly disordered. The numerous pores obstruct the diffusion of $\mathrm{Al}$ ions and further disturb ordering in alumina. Streaking and presence of forbidden reflections were attributed to stacking disorder. Such faults have also been observed in crystalline whiskers of $;$-alumina (Saraswati and Rao 1987). Peri (1965) modelled the surface of $\gamma$-alumina and found that the surface becomes more defective as the water molecules are removed beyond $80 \%$.

The absorption edge in the optical spectra for the undoped and sparely doped $(<1000 \mathrm{ppm}$ ) was at $5 \mathrm{eV}$, similar to amorphous alumina (Saraswati and Ramarao 1987). For the crystalline $\alpha$ alumina the absorption edge is at a higher energy $19 \mathrm{eV})$. Again, the IR absorption in the Al-O range of vibration frequency was similar to corundum in the $\alpha$ phase but was broad and weak in boehmite and transition aluminas (figure 2). Raman scattering was completely absent in the transition phases of alumina though a few peaks were seen for boehmite.

The band intensity criterion for IR and Raman is different. In IR, intensities depend on the magnitude of change in dipole moment during the course of vibration for a given mode. Therefore polar bonds give intense IR absorption. Asymmetric vibrations with greater dipole moment changes produce greater intensities. In Raman scattering, intensities depend in general on the change in polarizibility of bond during the course of vibration. So bonds with symmetric charge distribution and symmetric type of vibration give good intensities. In glasses where a coordination rule is maintained Raman bands are not affected. But in aluminosilicates the bands were weak and broad when the aluminium content was high (McMillan and Piriou 1982). These were attributed to either a range of coordination polyhedra, increasing in average coordination with increasing alumina content or, to geometric distortion and loss of vibrational coherence. Stacking disorder would introduce orientation-dependent distribution of dipole moment but would retain long range translational order. It must be mentioned here that there is a strong similarity between pseudoboehmite and clay, viz broad XRD lines, fine particle size, layered structure and swelling on adsorbing water, which affects the $d$ spacing of 001 reflections. Stacking models similar to clay could be adopted. Again, as boehmite is in a polymerized form, disorder at the chainfolds could also give rise to defects. 


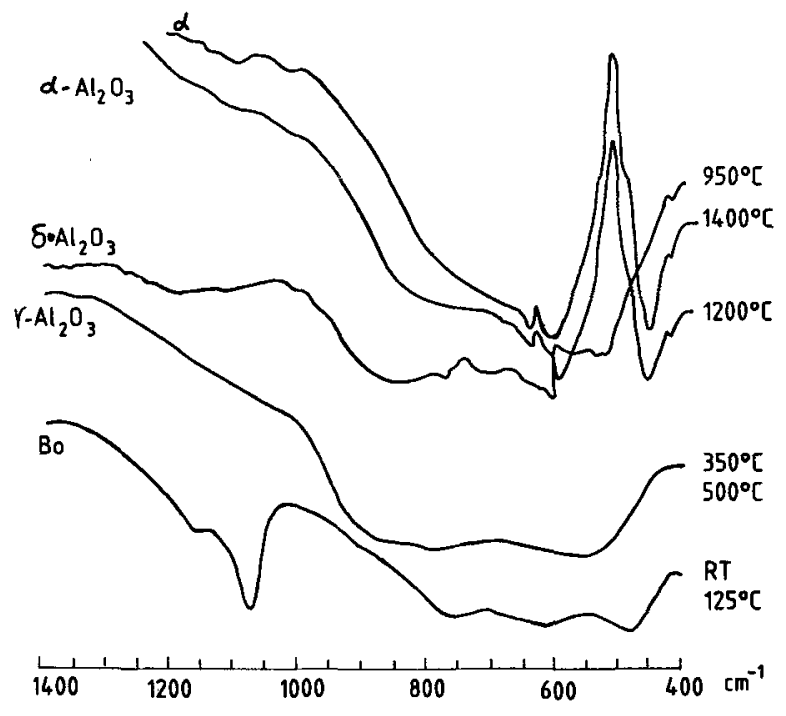

Figure 2. Infrared absorption in boehmite, transition aluminas and alpha alumina.

The xerogels of boehmite and transition aluminas are porous materials with nanometer size particles and large surface area, which result in large amount of unsaturated bonds and random distribution of charge. There is an evidence of disorder in charge distribution due to surface defects from NMR studies in $\gamma$ alumina (O'Reilly 1960). A nucleus with $I>\frac{1}{2}$ has quadrupole moment $Q$ which interacts with the electrical field gradient $q$ produced by an asymmetric distribution of charges around the nucleus. This interaction perturbs the magnetic resonance absorption. The resonance line frequency, shape and intensity are influenced depending on the strength of interaction. When the interaction is small, first order in $e^{2} q Q$, the central line is undisturbed and satellite line separation gives the coupling constant. When charge asymmetry is high, second order effects alter the position and shape of the central line. Its width or splitting estimates $e^{2} q Q$. In $\alpha$ alumina the $\mathrm{Al}^{27}(I=5 / 2)$ magnetic resonance showed second order quadrupolar broadening of the central line. $e^{2} q Q$ was found to be $2.32 \mathrm{MHz}$ and compared with corundum crystal. In $\gamma-\mathrm{Al}_{2} \mathrm{O}_{3}$ the intensity was very low (about 9/35 of total integrated intensity of $\alpha \mathrm{Al}_{2} \mathrm{O}_{3}$ ) and was found to decrease with increase in surface area linearly as $I=I_{0}(1-F)$ where $F$ is the fraction of ${ }^{27} \mathrm{Al}$ nuclei close to the surface of the solid which experiences large field gradients due to asymmetry in environment or defects. $\gamma-\mathrm{Al}_{2} \mathrm{O}_{3}$ has large surface area compared to $\alpha-\mathrm{Al}_{2} \mathrm{O}_{3}$ e.g. we observed $250 \mathrm{~m}^{2} / \mathrm{g}$ for $\gamma$ compared to $7 \mathrm{~m}^{2} / \mathrm{g}$ for $\alpha$. From the plot of intensity vs surface area it was estimated that on an average two layers of $\mathrm{Al}$ must be affected to wash out the NMR signal.

\section{Microstructure}

Some interesting features have been observed in the microstructures of boehmite and alumina xerogels (figure 3). Such features were observed in some organic 
polymers (Hull 1986) and $\mathrm{CuAl}_{2}-\mathrm{Al}$ eutectic alloy (Chadwick 1963) when solidified under unstable conditions. These are characteristics of solidifying and drying process rather than the material. Chalmers (1964) called these colony structures a combination of lamellar and cellular structure. These were said to be due to structure degeneration at very slow rates of solidification. Here the lamellae have widths $30-40 \mu \mathrm{m}$ (figure $3 \mathrm{a}$ ). Slow rates were used here for obtaining crack-free gels. Differing rates of diffusion either in the presence of impurities or mixed components could be the cause. The solidifying gel can be regarded as a two-component system consisting of polymerous boehmite and water, which evaporates out at a slow rate. The difference in viscosities, which is the driving force, makes one component (water) diffuse faster than the other and creates instabilities. This situation is similar to a Hele Shaw cell which is frequently used to simulate two fluids flow in a porous medium. The viscosity difference of two fluids in a laminar flow develops unstable flow displacements (figure $3 b$ ). Hence the colony structure observed could be regarded as a fractal structure. Self-similarity was checked by comparing the scanning electron micrographs at different magnification scales (figure 3c). Several wiggly lines were chosen from the micrographs and the number of steps required to traverse a line was plotted against the step size in a log scale. An object is a fractal if it is self-similar and obeys a power law equation, $n \propto r^{-D}$, where $n$ is the number of steps required to cover a wiggly line with steps of size $r . D$, the fractal dimension, is the slope of the straight line plot of $\log n$ vs $\log r$. An average of 5 slopes gave $D_{t}=1 \cdot 11+0 \cdot 01$. This indicates that irregularity is low or the fractal is smooth. Some error could arise if the lines have varying thickness. The surface fractal is related to line fractal as $D_{S}=D_{L}+1$ for isotropic objects (Mandelbrot 1982). Thus for alumina gel $D_{S}=1 \cdot 11+1=2 \cdot 11$, which would mean that the surface is not rough $(D=2$ for a smooth surface). Smooth surface fractals are found in layered structures (graphite), surface made of nanosize spherical particles and in molecular sieves like zeolites (Avnir et al 1983). Bochmite and transition aluminas satisfy the former situation. They have large surface areas and narrow pore distribution about $3 \mathrm{~nm}$. Sieve-like property is also possible and had been observed in chromia gel. This aspect is presently under investigation. Using the surface adsorption method with varied adsorbates, surface area in a porous alumina was found to be a fractal with dimension $D=2.79$ (Avnir et al 1984). When the particles are coarse their clustering would produce a rough surface and the, fractal dimension would show a higher irregularity.

\section{Phase separation}

Schaefer and Keefer (1984) postulated that phase separation should be observed in inorganic polymer gels similar to organic polymer gels (Tanaka 1978). We have observed such phase separation, viz regions of high and low polymer density in copper and chromium doped gels. Density fluctuations are seen on the top surface only when the gel is set to dry at a high viscosity. When a less concentrated solution is kept for drying no pattern appears. In figure 4 it appears as separation of polymeric and particulate forms. The Al-to-Cr ratio was alike in the two areas as seen from EDAX. $\gamma$-alumina whiskers grew out of $\mathrm{Cu}$-doped gel solution as a result of this phase separation. Though $\alpha$ alumina whiskers are common this is the first time $\gamma$ alumina crystals have grown without heat treatment. As mentioned earlier, 

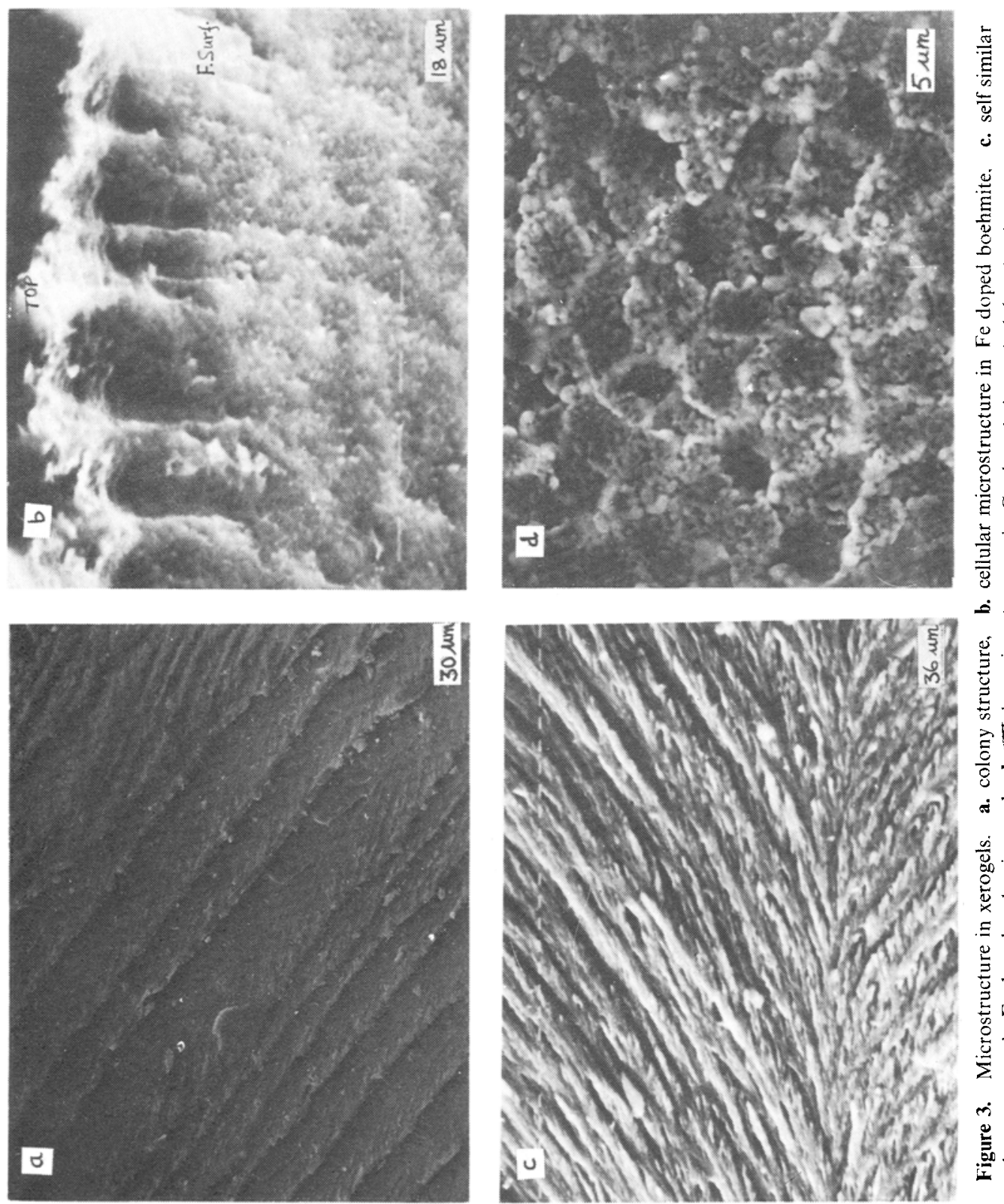

$\dot{5}$ 
stacking faults were also observed and the lattice parameter was higher than what was obtained earlier $(a=7-95$, spinel cube). This could be due to water bonded to the lattice. The whisker was soluble in water. On heating for $21 \mathrm{~h}$ at $500 \mathrm{C}$ no change in the diffraction was seen (Saraswati and Rao 1987).

\section{Toughness}

Owing to the ultrafine size of particles and pores the surface was smooth and glossy. Indentation hardness measurements could be carried out without any surface treatment despite high porosity $(60 \%)$. The Vickers hardness numbers followed the density changes and were related to the density by a power law (Saraswati 1987). Such a dependence can be expected for cellular materials. A plot of hardness against relative density in the log scale yields a slope $2 \cdot 4$. Cellular forms were observed on the top surface in Cu-doped gels in the $\alpha$ phase and also in Fedoped gel (figure 3). In the $\alpha$ phase, the Vickers hardness number was $960 \mathrm{~kg} / \mathrm{mm}^{2}$

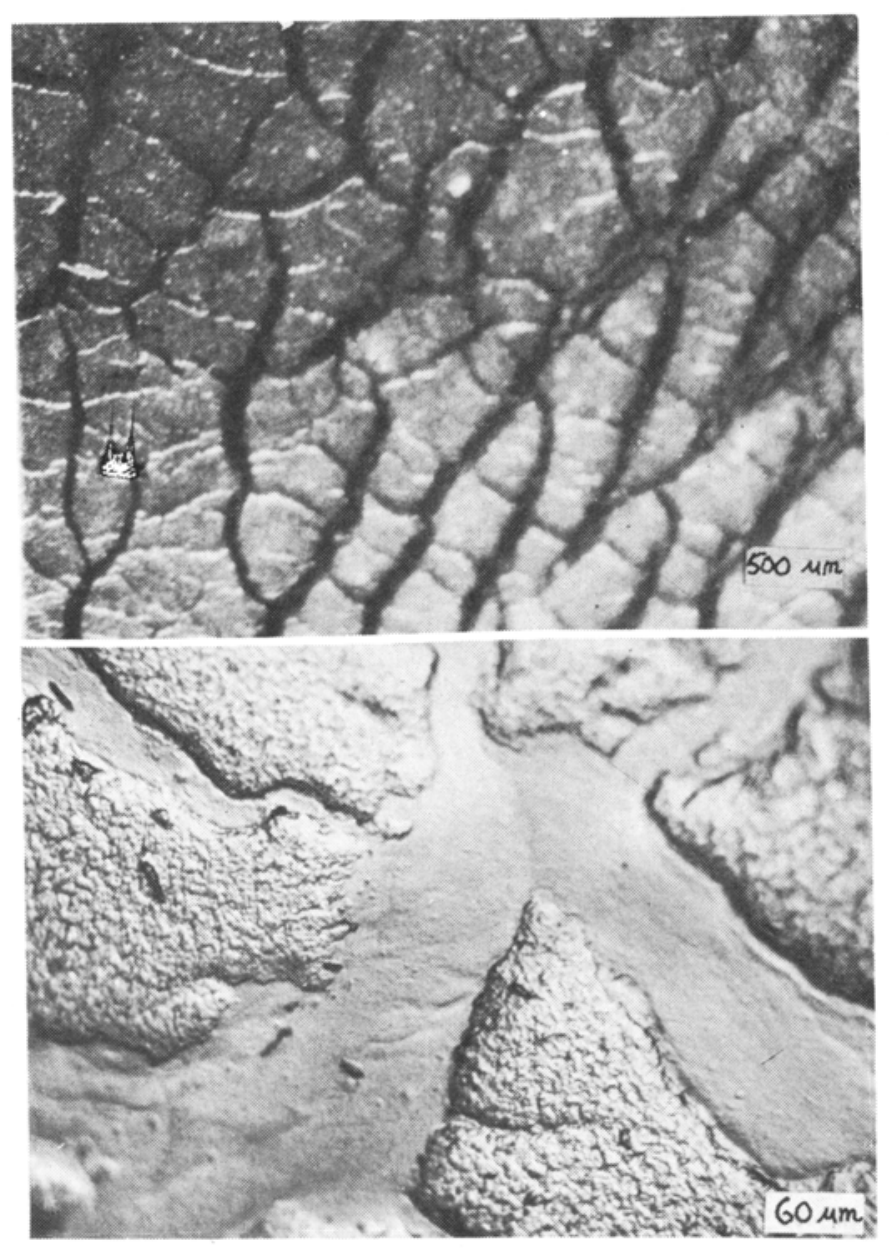

Figure 4. Phase separation in $\mathrm{Cr}$ doped monoliths. 
as against $1600 \mathrm{~kg} / \mathrm{mm}^{2}$ for corundum. But the impressions were crack-free even at $500 \mathrm{~g}$ load and flawless. This could be due to the increased toughness in the material.

\section{Conclusion}

It is concluded that the porous monoliths have some properties, viz optical absorption, microstructure and microhardness different from the usual alumina pellets. The difference arises due to the extremely fine size of particles, pores and cellular microstructure on account of slow drying which is required to produce a crack-free monolith of reasonable size. The absorption edge and cellular microstructure are similar to the amorphous anodized alumina. The monoliths are transparent only in the disordered phase. Hence they cannot replace pressure sintered crystalline transparent alumina. But the gel powder could be pressuresintered at a lower temperature for obtaining the same. The porous material could find use as filters and reactive supports.

\section{References}

Avnir D, Farin D and Pfeifer P 1983 J. Chem. Phys. 793566

Avnir D, Farin D and Pfeifer P 1984 Nature (London) 308261

Chadwick G A 1963 Prog. Mater. Sci. 1097

Chalmers B 1964 Principles in solidification (New York: John Wiley)

Hoffman D, Roy R and Komaneni S 1984 Mater. Sci. Lett. 2245

Hull D 1986 in Materials engineering (eds) J V Bee and G G Garett (London: Pergamon Press) p. 28

Lippens B C and deBoer J H 1964 Acta. Cryst. 171312

Mackenzie J D $1982 J$. Non-Cryst Solids 481

Mandelbrot B B 1982 The fractal geometry of nature (San Francisco: Freeman)

McMillan P and Piriou B 1982 J. Non-Cryst Solids 53279

O'Reilly D E 1960 Adv. Catal. 1231

Peri J B 1965 J. Phys. Chem. 69220

Pott G T and McNicol B D 1970 Chem. Phys. Lett. 6623

Roy R 1956 J. Am. Ceram. Soc. 39145

Roy R A and Roy R 1984 Mater. Res. Bull. 19169

Sakka S 1985 Am. Ceram. Soc. Bull. 641463

Saraswati V 1987 J. Mater. Sci. (in print)

Saraswati V and Ramarao G V 1987 Bull. Mater. Sci. 9193

Saraswati V and Rao G V N 1987 J. Cryst. Growth 83606

Saraswati V, Rao G V N and Ramarao G V 1987 J. Mater. Sci. 222529

Schaefer D W and Keefer K D 1984 Better ceramics through chemistry (eds) C J Brinker, D E Clark and D R Ulrich (New York: North Holland) p. 1

Tanaka T 1978 Phys. Rev. Lett. 40820

Uhlmann D R, Zelinski B J J and Wivek G E 1984 Better ceramics through chemistry (eds) C J Brinker, D E Clark and D R Ulrich (New York: North Holland)

Wilson S J 1979 J. Solid State Chem. 30257

Yoldas B E 1975 Am. Ceram. Soc. Bull. 54289

Yoldas B E 1982 J. Non-Cryst. Solids 51105 